\title{
Digestive
}

Dig Dis 2017;35:251-258

DOI: $10.1159 / 000450983$

\section{Bile Acid Uptake Transporters as Targets for Therapy}

\author{
Davor Slijepcevic ${ }^{a}$ Stan F.J. van de Graafa, b

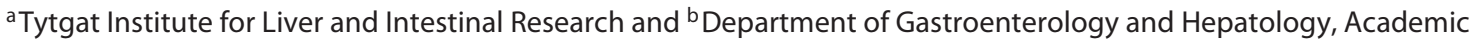 \\ Medical Center, Amsterdam, The Netherlands
}

\section{Keywords}

Sodium taurocholate cotransporting polypeptide .

Apical sodium-dependent bile acid transporter .

Bile acid signaling $\cdot$ Hepatitis B virus $\cdot$ Cholestasis

\begin{abstract}
Background: Bile acids are potent signaling molecules that regulate glucose, lipid and energy homeostasis predominantly via the bile acid receptors farnesoid $X$ receptor (FXR) and transmembrane $G$ protein-coupled receptor 5 (TGR5). The sodium taurocholate cotransporting polypeptide (NTCP) and the apical sodium dependent bile acid transporter (ASBT) ensure an effective circulation of (conjugated) bile acids. The modulation of these transport proteins affects bile acid localization, dynamics and signaling. The NTCP-specific pharmacological inhibitor myrcludex B inhibits hepatic uptake of conjugated bile acids. Multiple ASBTinhibitors are already in clinical trials to inhibit intestinal bile acid uptake. Here, we discuss current insights into the consequences of targeting bile acid uptake transporters on systemic and intestinal bile acid dynamics and discuss the possible therapeutic applications that evolve as a result.
\end{abstract}

(c) 2017 The Author(s)

Published by S. Karger AG, Basel

\section{KARGER}

E-Mail karger@karger.com www.karger.com/ddi
This article is licensed under the Creative Commons AttributionNonCommercial-NoDerivatives 4.0 International License (CC BYNC-ND) (http://www.karger.com/Services/OpenAccessLicense) Usage and distribution for commercial purposes as well as any

\section{Introduction}

Bile acid transport into the liver and intestine is maintained by transport proteins, present at the apical membrane of ileocytes and basolateral membrane of hepatocytes. In this review, we focus on hepatic bile acid uptake via sodium taurocholate cotransporting polypeptide (NTCP) and intestinal bile acid uptake via apical sodium-dependent bile acid transporter (ASBT) and the (possible) benefits of pharmacologically targeting these transporters in various pathophysiological conditions. First, the discovery of NTCP as the key uptake receptor for hepatitis $\mathrm{B}$ and $\mathrm{D}$ virus (HBV/HDV) pointed to novel applications of NTCP targeting in virology. Second, physiological and pharmacological downregulation of the hepatic and intestinal bile acid uptake machinery during cholestasis might provide hepatoprotection. Third, the modulation of bile acid transport is expected to alter bile acid dynamics, which in turn will affect the activation of the main bile acid sensors farnesoid X receptor (FXR) and transmembrane G proteincoupled receptor 5 (TGR5), with multiple consequences on metabolism of bile acids, cholesterol, lipids and glucose.

Stan F.J. van de Graaf

Tytgat Institute for Liver and Intestinal Research

Department of Gastroenterology and Hepatology, Academic Medical Center

NL-1105 BK Amsterdam (The Netherlands)

E-Mail k.f.vandegraaf@amc.uva.nl 


\section{Bile Acid Formation and the Enterohepatic Circulation}

Bile excretion plays a pivotal role in the elimination of endogenous and exogenous (toxic) compounds, such as bilirubin, heavy metals and drug metabolites. Bile mainly contains phospholipids, cholesterol and bile acids combined in micelles [1]. Bile acids are amphipathic molecules, characterized by the ability to form an interface between lipids and water. In the small intestine, bile acids improve dietary lipid digestion and transport across enterocytes.

Synthesis of bile acids starts in the pericentral hepatocytes via the rate-limiting conversion of cholesterol into $7 a$-hydroxycholesterol by the hepatic microsomal enzyme cholesterol 7a-hydroxylase (CYP7A1). After a complex biosynthetic pathway involving multiple enzymes, the main primary bile acids chenodeoxycholic acid (CDCA) and cholic acid (CA) are formed ( $80 \%$ of the human bile acid pool) [2]. Specifically in mice, CDCA is converted to muricholic acid. In the last step of biosynthesis, bile acids undergo conjugation with glycine (predominantly in humans) or with taurine (predominantly in rodents). Secreted bile acids are predominantly in their conjugated form, that is, in the negatively charged state, which prevents passive membrane-diffusion. Secondary bile acids are formed from these primary bile acids by bacterial modification in the distal intestine [3]. Bile acids are efficiently retained within the enterohepatic circulation (only $3-5 \%$ is lost via feces), due to reabsorption in the terminal ileum by the apical sodium-dependent bile acid transporter (SLC10A2/ASBT) and excretion into portal blood by the heterodimeric organic solute transporter $\alpha / \beta$ $(S L C 51 A / B / O S T \alpha / \beta)$ and other basolateral extrusion pathways in ileocytes $[4,5]$. In physiological situations, fecal bile acid loss is compensated for by de novo synthesis from cholesterol in the liver, and thereby boosts cholesterol elimination from the body [6]. Bile acids are eventually recycled from portal blood at the hepatic basolateral membrane by 2 transport systems: the sodium-dependent taurocholate cotransporting polypeptide (SLC10A1/ NTCP) and members of the sodium-independent organic anion transporting polypeptide (SLCO/OATP) transport family $[7,8]$. In humans, the physiological bile acid concentration ranges from $<5 \mu \mathrm{M}$ (fasting) to $3-7 \mu \mathrm{M}$ (postprandial) in systemic blood. In portal blood, the postprandial bile acid peak is more evident (from 4-27 to 22$55 \mu \mathrm{M})[9,10]$. Thus, the hepatic uptake machinery efficiently limits the escape of bile acids to the general circulation, with a first-pass extraction fraction ranging from 50 to $90 \%$ depending on the bile acid structure [11].

\section{Hepatic Basolateral Uptake Systems}

NTCP is a family member of the solute carrier 10 family and is present on the basolateral membrane of hepatocytes. NTCP adopts a dimeric or even higher order quaternary structure, in which the individual subunits form functional units [12]. Recently, the crystal structure of ASBT was solved revealing a structure with 9 transmembrane domains, with an exoplasmic $\mathrm{N}$ terminus and cytoplasmic $\mathrm{C}$ terminus [13], and a similar conformation might be expected for NTCP. NTCP-mediated uptake of taurocholate was demonstrated with published $\mathrm{K}_{\mathrm{m}}$ values that vary from 5 to $84 \mu \mathrm{M}$ for human NTCP and 8-61 $\mu \mathrm{M}$ for rodent NTCP [14]. Besides bile acids, NTCP is a transporter of steroidal hormones and a variety of drugs [15, 16]. In the liver, NTCP is distributed equally along all liver lobules, but uptake of conjugated bile acids occurs predominantly in periportal cells (zone 1), as these are exposed to the highest concentrations of bile acids [17]. In humans, Ho et al. [18] described ethnicity-dependent polymorphisms that are associated with decreased transport function in vitro. However, to date, only one individual with NTCP deficiency was described, featuring a single homozygous mutation (p.R252H), phenotypically characterized by high plasma conjugated bile acid levels without any signs of liver injury or pruritus [19]. Similarly, a subset of Slc10a1/NTCP knockout mice displays strongly elevated conjugated bile acids levels in plasma [20], confirming a primary role for NTCP in hepatic clearance of conjugated bile acids. In addition, several adult NTCP knockout mice showed physiological bile acid levels, indicating that an NTCP-independent uptake of conjugated bile acids must exist.

The presence of additional $\mathrm{Na}^{+}$-dependent membrane transporters has been suggested, in particular, bile acid transport by microsomal epoxide hydrolase [21], but little experimental evidence supports this notion [22]. Hepatic bile acid uptake can also be mediated by (one or more members of) the $\mathrm{Na}^{+}$-independent OATP transporter family. All OATPs are 12 transmembrane domain glycoproteins with broad substrate preference, such as (un)conjugated bile acids, bilirubin and numerous drugs [23]. The most abundant hepatic OATP subfamilies are OATP1A and OATP1B, and are designated Oatp1a1, Oatp1a4 and Oatp1b2 in rodents [8]. It is difficult to estimate the role of each single OATP-isoform in vivo, as there is large substrate overlap and rodent Oatps have no direct human orthologue [24]. In humans, 2-gene biallelic human OATP1B1 and OATP1B3 deficiency is known as the Rotor syndrome, character-
252

Dig Dis 2017;35:251-258 DOI: $10.1159 / 000450983$
Slijepcevic/van de Graaf 
Fig. 1. NTCP and ASBT: topology and ligands. Both NTCP (upper panel) and ASBT (lower panel) from the SLC10 family contain 9 transmembrane spanning domains. Conjugated bile acids are natural substrates for these transporters, and recently the preS1 domain of HBV was found to specifically bind to NTCP. Also, the HBV-derived lipopeptide myrcludex B strongly binds to and inhibits NTCP in vitro and in vivo. Many ASBT-specific inhibitors (e.g. A4250) were developed over the past decade to increase fecal bile acid excretion.

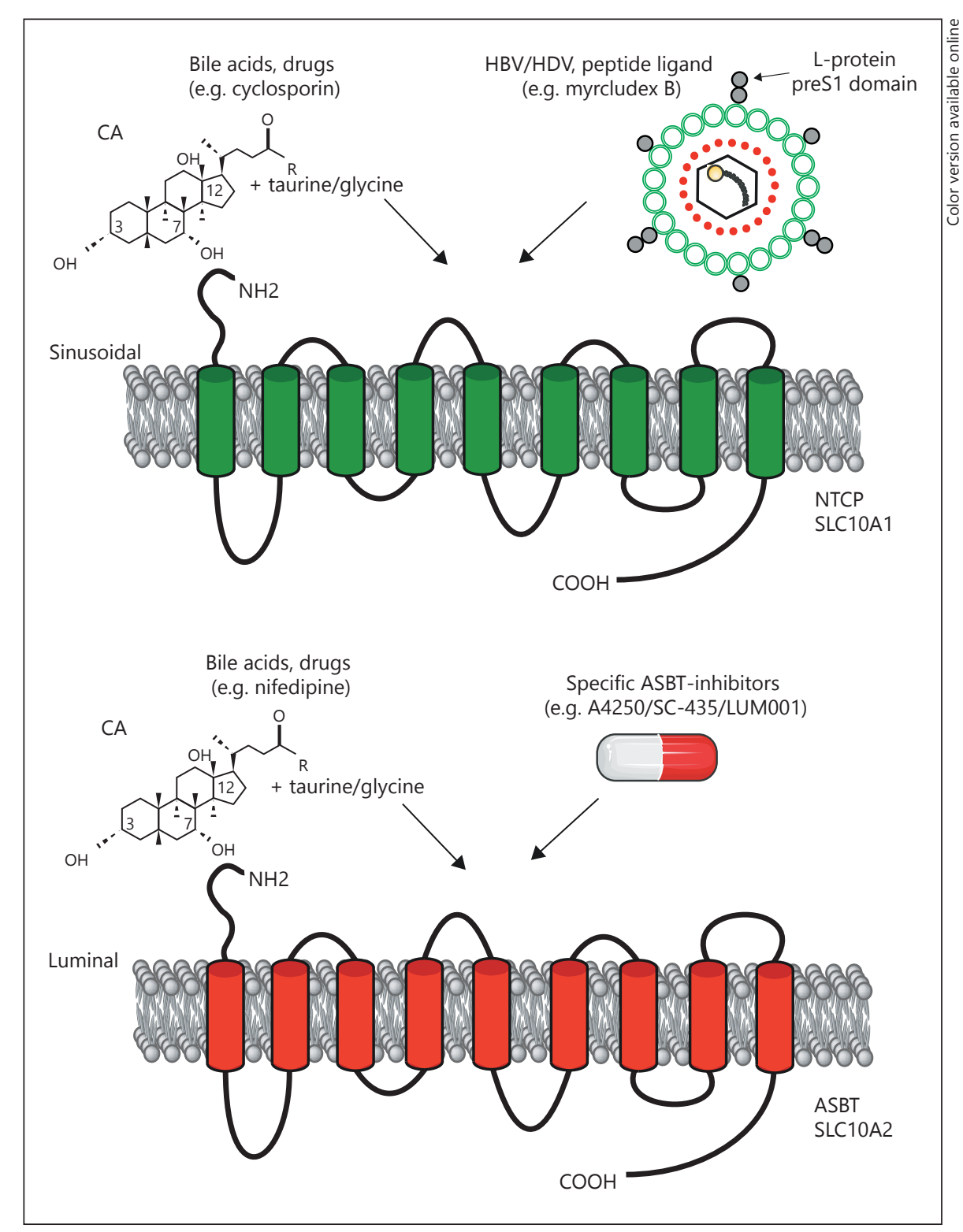

ized by high conjugated plasma bilirubin levels [25]. Evidence for human OATP polymorphisms affecting endogenous bile acid uptake is sparse. Interestingly, mice lacking all Oatp1a/1b-family members (Slco 1a/1b knockout mice) display 13-fold elevated levels of unconjugated bile acid in blood [26], whereas conjugated bile acid levels remained mostly unchanged, and similar results were found in knockout mice lacking only Slco 1 b2 [27]. The role of the rodent Oatp1a-isoforms in bile acid transport is less obvious; nevertheless, taurocholate uptake was reduced in primary hepatocytes isolated from Oatp1a4-null mice, and to slighter extent in Oatpla1null mice [28]. Other studies suggest that Oatpla1 and

Bile Acid Uptake Transporters as Targets for Therapy
Oatp1a4 preferably transport secondary unconjugated bile acids, thereby altering intestinal bile acid metabolism $[29,30]$.

\section{NTCP as Receptor of HBV/HDV}

Beside the role of NTCP as major transporter for conjugated bile acids, NTCP was recently found to be the main receptor for HBV and HDV viral particles [31], and the specific NTCP inhibitor myrcludex B is currently being tested in phase II trials as an HBV/HDV entry inhibitor [32, 33] (fig. 1). Myrcludex B is a synthetic lipopep- 
tide based on the preS1 domain of the HBV envelop protein targeting NTCP, and effectively inhibits HBV entry in vitro and in vivo $[34,35]$. Pharmacokinetic studies with myrcludex B show rapid hepatic accumulation (within minutes) [20] where it has a half-life of $\sim 12 \mathrm{~h}$. Liver-specific binding is also observed in non-HBV susceptible animals (dogs, rats and mice) [36] and NTCP specificity was recently confirmed using NTCP knockout mice [20]. $\mathrm{HBV}$ entry inhibition is clinically important in the treatment of HBV/HDV infection, where it could be applied in combination with other anti-viral drugs, such as interferon or tenefovir. An interim report of a phase Ib/IIa clinical trial showed a reduction of HDV RNA levels towards undetectable levels upon 12 or 24 weeks of treatment especially using a combination of myrcludex B and interferon, although $\mathrm{HBsAg}$ did not show any reduction with this treatment duration [32]. Myrcludex B treatment resulted in elevated plasma bile acids levels, which was well tolerated and the drug showed no adverse effects.

\section{Inhibition of Bile Acid Uptake to Ameliorate Cholestatic Liver Injury}

Cholestatic liver damage occurs when bile flow is impeded, leading to the accumulation of toxic bile acids within hepatocytes and causing liver damage, inflammation and fibrosis. Current therapies in cholestatic liver disease are recently reviewed by Beuers et al. [37]. Nuclear receptors (NRs), bile acid transporters and hepatic enzymes play a key role in orchestrating bile acid metabolism to protect against the accumulation of toxic bile acids [38]. Classical intrahepatic bile acid sensing by FXR [3941] leads to the recruitment of the atypical NR that has only a ligand-binding domain, called short heterodimer partner (SHP) [42, 43], which represses bile acid biosynthesis via the downregulation of CYP7A1. Hepatic FXR activation by (semi-)synthetic FXR agonists has been successful in cholestatic animal models $[44,45]$ in order to induce hepatic bile acid efflux and reduce bile acid uptake and such therapies are now being tested in phase II and III trials in PBC and primary sclerosing cholangitis [46]. In addition to FXR-dependent transcriptional repression of the bile acid uptake machinery, posttranscriptional regulation of the plasma membrane expression and function of NTCP is expected to be relevant during cholestasis, as reviewed elsewhere [47]. So far, the effectiveness of (further) inhibition of basolateral hepatic bile acid uptake in the context of cholestasis was only studied using OATP1A1 knockout mice, which were not protected against hepatic injury after bile-duct ligation [48]. The role of additional (pharmacological) NTCP-inhibition during cholestasis is not reported yet. The finding that myrcludex B is well tolerated, even at dosages that inhibit NTCP and lead to increased bile acid levels in plasma, makes this strategy likely to be tested in vivo.

When we focus on the modulation of intestinal bile acid transport, most studies investigated intestinal bile acid sequestrants (e.g. cholestyramine and colesevelam) as first-line agents in the treatment of cholestatic pruritis. Colesevelam proved to be effective in reducing plasma bile acid levels by $\sim 50 \%$, but failed to reduce pruritis [49]. Currently, clinical studies are evaluating ASBT inhibitors as a novel pharmacological treatment for cholestasis. The rational of ASBT inhibition is based on an increase of fecal bile acid elimination and preventing bile acid return to the liver, thereby potentially reducing the bile acid pool by $80 \%$. The ASBT inhibitor A4250, previously used in a clinical phase I study [50], showed improvement of cholestatic liver injury in multi-drug resistance P-glycoprotein 2-deficient mice [51]. In a similar study, 2 weeks of treatment with SC-435, a different ASBT inhibiting small molecule, demonstrated reduced bile acid pool size and attenuation of cholestasis in the same mouse model [52]. At present, human studies are being performed, but the outcome of these promising studies is not published yet (https://clinicaltrials.gov/ct2/show/NCT02061540).

Targeting FXR tissue-specifically during cholestasis attracted considerable attention since the discovery of fibroblast growth factor 15/19 (FGF15/19). This hormone, when released from the gut, binds to the tyrosine kinase receptor FGF receptor $4 / \beta$-Klotho on hepatocytes, which activates the jun $\mathrm{N}$-terminal kinase $1 / 2$ signaling pathway [53]. Intestinal FXR activation [54] and the FGF19 mimetic M70 [55, 56] dampen cholestatic liver injury by strongly reducing hepatic bile acid synthesis and the circulating bile acid pool. So, mouse studies clearly show hepatoprotection through (gut-specific) FGF15/19 signaling, primarily by reducing bile acid pools, sharing its mode of action with ASBT-inhibition. NGM282 (the engineered variant of human FGF19) is now tested in a phase II trial in PBC patients unresponsive to ursodeoxycholic acid treatment [57].

\section{Bile Acid Dynamics in Relation to the Metabolic State}

Besides the role of bile acids in cholesterol and lipid metabolism, bile acids are important signaling molecules regulating glucose metabolism, inflammation and energy
254

Dig Dis 2017;35:251-258 DOI: $10.1159 / 000450983$
Slijepcevic/van de Graaf 
Fig. 2. Enterohepatic circulation of bile acids. a Overview of the distinct hepatic and intestinal bile acid transporters. The key transporters are BSEP, ASBT, OST $\alpha / \beta$, OATP and NTCP (schematically depicted). Upon intracellular bile acid sensing, FXR/SHP and FGF15/19 become activated and regulate bile acid synthesis (i.e. predominantly CYP7A1). Furthermore, spillover of bile acids into the systemic circulation might also activate the TGR5 receptor, present on the basolateral side in various tissues, such as BAT, muscle and in the colon. b Pharmacological inhibition of ileal bile acid uptake (by bile acid binding resins or ASBT inhibitors) induces the presence of bile acids in the colon and increases fecal bile acid loss. The latter contributes to cholesterol catabolism. Increased GLP1 release in the distal intestine improves systemic glucose handling and is likely induced by (passive) the translocation of bile acids leading to basolateral stimulation of TGR5.

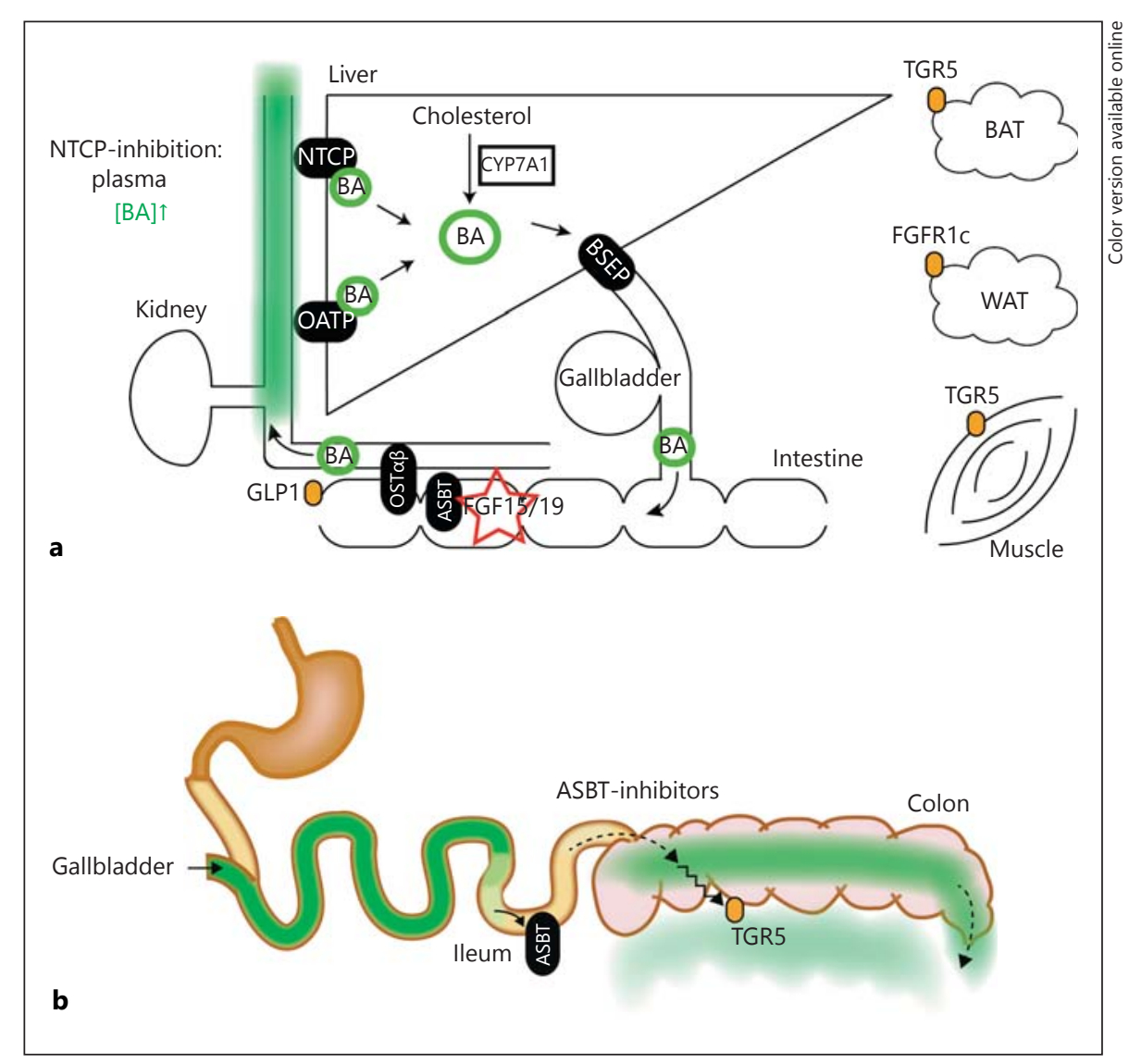

expenditure. Detection of bile acids is mostly mediated via the receptors FXR and TGR5, and not exclusively in the liver and intestine $[58,59]$. Targeting bile acid signaling is, therefore, appealing to treat metabolic diseases such as diabetes and atherosclerosis. In the following sections, we discuss the (possible) beneficial metabolic effects of modulation of bile acid dynamics by the inhibition of ASBT and NTCP transport activity as this is expected to alter the FXR/TGR5 activity.

Inhibition of intestinal bile acid uptake specifically via ASBT is recognized for its low-density lipoprotein (LDL)cholesterol lowering effect [60]. Preclinical studies further showed that ASBT-inhibition reduces hepatic triglyceride and cholesterol accumulation in high fat dietfed mice [61, 62]. Similarly, bile acid-binding resins, including cholestyramine and colesevelam, have been shown to reduce serum total sterols including LDL-cholesterol values. This effect is mainly due to increase in cholesterol catabolism to replenish bile acids lost via the feces. However, their use for this application is limited, mainly because compliance remains a challenge due to the gastrointestinal side effects.

Bile Acid Uptake Transporters as Targets for Therapy
Bile acid-binding resins also have a beneficial effect on glucose handling. For example, the bile-acid sequestrant Colestilan induces glucagon-like peptide 1 (GLP1) release from the colon [63]. GLP1 increases insulin secretion and improves insulin sensitivity. Similarly, colesevelam improved glucose homeostasis. This effect seems mostly mediated by TGR5 activation [63, 64], and partially through the inhibition of FXR signaling [65]. Bile acid-induced GLP1 release occurs upon the activation of TGR5 at the basolateral (blood) side of L-cells $[66,67]$. Nevertheless, ASBT inhibitors and bile acid-binding resins both stimulate the release of enterohepatic hormones, including GLP-1, and inhibit the uptake of bile acid into the circulation. In our view, the most direct explanation for this apparent discrepancy is that TGR5-mediated effects occur after (passive) bile acid translocation to the basolateral side of the colonocyte (fig. 2). ASBT-inhibition and bile-acid sequestration result in the increased presence of bile acid in the colon. Bile acid sequenstrants do not covalently bind bile acids, and some diffusion is still likely. The secondary bile acids lithocholic and deoxycholic acid, (and their corresponding taurine and gly-

Dig Dis 2017;35:251-258 DOI: $10.1159 / 000450983$ 
cine conjugates) are most potent activators of TGR5 in vitro, so local elevation of these bile acid species could stimulate GLP-1 release into the circulation. At present, it is unclear whether elevated bile acid levels in the systemic circulation also would stimulate GLP1-secretion. Thomas et al. [68] demonstrated that the positive effects on glucose homeostasis of the CA-derived TGR5 agonist INT-777 were also mediated by intestinal TGR5. However, INT-777 was provided orally in this study, so TGR5 might have been activated from either the systemic circulation or by local diffusion across the colonic epithelium.

In general, little is known about the influence of endogenous circulating bile acids on metabolic processes, and whether inhibition of hepatic bile acid (re)uptake boosts energy expenditure and/or lipid metabolism. The activation of TGR 5 by bile acids is linked to increased energy expenditure in brown adipose tissue (BAT) and muscle [69]. We postulate that such effects are mimicked by (transiently) increased bile acids levels that would occur with NTCP inhibition. This process could also play a role in the beneficial metabolic consequences of bariatric surgery. Bariatric procedures might induce bile-acid signaling by increased circulating bile acid levels, as shown in several studies [70, 71]. Previously, ileal interposition surgery in mice showed strongly elevated plasma bile acid levels [72], without changes in hepatic Ntcp mRNA. Beneficial effects of vertical sleeve gastrectomy (VSG) on body weight and glucose tolerance are dependent on both FXR [73] and TGR5 [74], suggesting that bile acid signaling is indeed relevant. Interestingly, mRNA-seq in VSG mice showed significant downregulation of hepatic Ntcp and Oatp1b2, possibly explaining the increase in bile acid levels in plasma [75].

What happens to the liver when NTCP is inhibited and/or bile acid levels in the systemic circulation increase? TGR5 is not detected in hepatocytes, but is expressed in sinusoidal endothelial cells where its activation has a hepatoprotective role by inducing nitric oxide synthase in a cAMP-dependent manner [76]. Furthermore,
TGR5 activation in Kupffer cells decreases the release of proinflammatory cytokines IL- 6 and TNF- $\alpha[77,78]$ and (more generally) dampens macrophage-mediated inflammation by inhibiting the NFkB-pathway [79]. A high-fat diet caused more liver steatosis in male TGR5knockout mice [80], suggesting that TGR5 activation prevents non-alcoholic fatty liver disease. INT-777 reduces liver fatty acid and triglyceride content as well as plasma triglycerides [68]. A beautiful study using glucocorticoid receptor-deficient mice pointed to a contribution of NTCP-governed bile acid dynamics to metabolism [81]. These mice showed impaired hepatic bile acid uptake by the downregulation of NTCP, which reduces dietary fat absorption and increases BAT mitochondrial uncoupling. Reduced bile acid uptake via NTCP could also dampen hepatic FXR activation. The (metabolic) consequences of this action are currently not clear, although some first insights were obtained using a mouse model, where human hepatocytes repopulated the liver of urokinase plasminogen activator/severe combined immunodeficiency mice. NTCP inhibition using myrcludex B resulted in increased CYP7A1 expression, suggesting reduced FXR activity [82]. Chronic HBV infection in these humanized mice had a similar effect. Further studies are required to assess the (metabolic) consequences of NTCP inhibition, as effects on lipid, glucose or energy metabolism were not investigated in this study.

In summary, targeting bile acid uptake transporters, NTCP and ASBT, has exciting implications for the fields of virology, cholestasis and metabolism of glucose, lipid and energy, although therapeutic efficacy and long-term (side-)effects of altering the bile acid dynamics needs to be further elucidated.

\section{Disclosure Statement}

The authors have nothing to disclose.

\section{References}

1 Esteller A: Physiology of bile secretion. World J Gastroenterol 2008;14:5641-5649.

12 Russell DW: The enzymes, regulation, and genetics of bile acid synthesis. Annu Rev Biochem 2003;72:137-174.

-3 Hofmann AF, Sjövall J, Kurz G, et al: A proposed nomenclature for bile acids. J Lipid Res 1992;33:599-604.

4 Hofmann AF: The enterohepatic circulation of bileacidsin mammals: form and functions. Front Biosci (Landmark Ed) 2009;14:2584-2598.
5 Dawson PA, Karpen SJ: Intestinal transport and metabolism of bile acids. J Lipid Res 2015; 56:1085-1099.

6 Vlahcevic ZR, Pandak WM, Stravitz RT: Regulation of bile acid biosynthesis. Gastroenterol Clin North Am 1999;28:125.

7 Kullak-Ublick GA, Stieger B, Meier PJ: Enterohepatic bile salt transporters in normal physiology and liver disease. Gastroenterology 2004;126:322-342.
8 Meier PJ, Stieger B: Bile salt transporters. Annu Rev Physiol 2002;64:635-661.

-9 Angelin B, Björkhem I, Einarsson K, et al: Hepatic uptake of bile acids in man. Fasting and postprandial concentrations of individual bile acids in portal venous and systemic blood serum. J Clin Invest 1982;70:724-731.

10 Linnet K: Postprandial plasma concentrations of glycine and taurine conjugated bile acids in healthy subjects. Gut 1983;24:249252. 
11 Meier PJ: Molecular mechanisms of hepatic bile salt transport from sinusoidal blood into bile. Am J Physiol 1995;269(6 pt 1):G801G812.

12 Bijsmans IT, Bouwmeester RA, Geyer J, et al: Homo- and hetero-dimeric architecture of the human liver $\mathrm{Na}+$-dependent taurocholate co-transporting protein. Biochem J 2012;441: 1007-1016.

$13 \mathrm{Hu}$ NJ, Iwata S, Cameron AD, et al: Crystal structure of a bacterial homologue of the bile acid sodium symporter ASBT. Nature 2011; 478:408-411.

14 Stieger B: The role of the sodium-taurocholate cotransporting polypeptide (NTCP) and of the bile salt export pump (BSEP) in physiology and pathophysiology of bile formation. Handb Exp Pharmacol 2011;201:205-259.

15 Kim RB, Leake B, Cvetkovic M, et al: Modulation by drugs of human hepatic sodium-dependent bile acid transporter (sodium taurocholate cotransporting polypeptide) activity. J Pharmacol Exp Ther 1999;291:1204-1209.

16 Visser WE, Wong WS, van Mullem AA, et al: Study of the transport of thyroid hormone by transporters of the SLC10 family. Mol Cell Endocrinol 2010;315:138-145.

17 Groothuis GM, Hardonk MJ, Keulemans KP, et al: Autoradiographic and kinetic demonstration of acinar heterogeneity of taurocholate transport. Am J Physiol 1982;243:G455G462.

18 Ho RH, Leake BF, Roberts RL, et al: Ethnicitydependent polymorphism in Na+-taurocholate cotransporting polypeptide (SLC10A1) reveals a domain critical for bile acid substrate recognition. J Biol Chem 2004;279:72137222 .

19 Vaz FM, Paulusma CC, Huidekoper H, et al: Sodium taurocholate cotransporting polypeptide (SLC10A1) deficiency: conjugated hypercholanemia without a clear clinical phenotype. Hepatology 2015;61:260-267.

20 Slijepcevic D, Kaufman C, Wichers CG, et al: Impaired uptake of conjugated bile acids and hepatitis b virus pres1-binding in na(+) -taurocholate cotransporting polypeptide knockout mice. Hepatology 2015;62:207-219.

-21 Von Dippe P, Amoui M, Alves C, et al: $\mathrm{Na}(+)$ dependent bile acid transport by hepatocytes is mediated by a protein similar to microsomal epoxide hydrolase. Am J Physiol 1993; 264(3 pt 1):G528-G534.

22 Honscha W, Platte HD, Oesch F, et al: Relationship between the microsomal epoxide hydrolase and the hepatocellular transport of bile acids and xenobiotics. Biochem J 1995; 311(pt 3):975-979.

-23 Kullak-Ublick GA, Stieger B, Hagenbuch B, et al: Hepatic transport of bile salts. Semin Liver Dis 2000;20:273-292.

24 Klaassen CD, Aleksunes LM: Xenobiotic, bile acid, and cholesterol transporters: function and regulation. Pharmacol Rev 2010;62:1-96.

-25 van de Steeg E, Stránecký V, Hartmannová H, et al: Complete OATP1B1 and OATP1B3 deficiency causes human Rotor syndrome by in- terrupting conjugated bilirubin reuptake into the liver. J Clin Invest 2012;122:519-528.

26 van de Steeg E, Wagenaar E, van der Kruijssen $\mathrm{CM}$, et al: Organic anion transporting polypeptide 1a/1b-knockout mice provide insights into hepatic handling of bilirubin, bile acids, and drugs. J Clin Invest 2010;120:29422952.

27 Csanaky IL, Lu H, Zhang Y, Ogura K, et al: Organic anion-transporting polypeptide 1b2 (Oatp1b2) is important for the hepatic uptake of unconjugated bile acids: Studies in Oatp1b2-null mice. Hepatology 2011;53:272281.

28 Gong L, Aranibar N, Han YH, et al: Characterization of organic anion-transporting polypeptide (Oatp) 1a1 and 1a4 null mice reveals altered transport function and urinary metabolomic profiles. Toxicol Sci 2011;122: 587-597.

29 Zhang Y, Csanaky IL, Selwyn FP, et al: Organic anion-transporting polypeptide la4 (Oatpla4) is important for secondary bile acid metabolism. Biochem Pharmacol 2013; 86:437-445.

-30 Zhang Y, Csanaky IL, Lehman-McKeeman $\mathrm{LD}$, et al: Loss of organic anion transporting polypeptide 1al increases deoxycholic acid absorption in mice by increasing intestinal permeability. Toxicol Sci 2011;124:251-260.

31 Yan H, Zhong G, Xu G, et al: Sodium taurocholate cotransporting polypeptide is a functional receptor for human hepatitis $\mathrm{B}$ and $\mathrm{D}$ virus. Elife 2012;1:e00049.

- 32 Bogomolov P, Alexandrov A, Voronkova N, et al: Treatment of chronic hepatitis D with the entry inhibitor myrcludex B: first results of a phase Ib/IIa study. J Hepatol 2016;65: 490-498.

33 Blank A, Markert C, Hohmann N, et al: Firstin-human application of the novel hepatitis B and hepatitis D virus entry inhibitor myrcludex B. J Hepatol 2016;65:483-489.

34 Schulze A, Schieck A, Ni Y, et al: Fine mapping of pre-S sequence requirements for hepatitis $B$ virus large envelope protein-mediated receptor interaction. J Virol 2010;84:19892000.

35 Volz T, Allweiss L, Ben MBarek M, et al: The entry inhibitor Myrcludex-B efficiently blocks intrahepatic virus spreading in humanized mice previously infected with hepatitis B virus. J Hepatol 2013;58:861-867.

36 Schieck A, Schulze A, Gähler C, et al: Hepatitis $B$ virus hepatotropism is mediated by specific receptor recognition in the liver and not restricted to susceptible hosts. Hepatology 2013;58:43-53.

37 Beuers U, Trauner M, Jansen P, et al: New paradigms in the treatment of hepatic cholestasis: from UDCA to FXR, PXR and beyond. J Hepatol 2015;62(1 suppl):S25S37.

38 Eloranta JJ, Kullak-Ublick GA: Coordinate transcriptional regulation of bile acid homeostasis and drug metabolism. Arch Biochem Biophys 2005;433:397-412.
Makishima M, Okamoto AY, Repa JJ, et al: Identification of a nuclear receptor for bile acids. Science 1999;284:1362-1365.

40 Parks DJ, Blanchard SG, Bledsoe RK, et al: Bile acids: natural ligands for an orphan nuclear receptor. Science 1999;284:1365-1368.

41 Wang H, Chen J, Hollister K, et al: Endogenous bile acids are ligands for the nuclear receptor FXR/BAR. Mol Cell 1999;3:543553.

42 Goodwin B, Jones SA, Price RR, et al: A regulatory cascade of the nuclear receptors FXR, SHP-1, and LRH-1 represses bile acid biosynthesis. Mol Cell 2000;6:517-526.

43 Lu TT, Makishima M, Repa JJ, et al: Molecular basis for feedback regulation of bile acid synthesis by nuclear receptors. Mol Cell 2000;6: 507-515.

44 Pellicciari R, Fiorucci S, Camaioni E, et al: 6alpha-ethyl-chenodeoxycholic acid (6-ECDCA), a potent and selective FXR agonist endowed with anticholestatic activity. J Med Chem 2002; 45:3569-3572.

45 Liu Y, Binz J, Numerick MJ, et al: Hepatoprotection by the farnesoid $\mathrm{X}$ receptor agonist GW4064 in rat models of intra- and extrahepatic cholestasis. J Clin Invest 2003;112:16781687.

46 Hirschfield GM, Mason A, Luketic V, et al: Efficacy of obeticholic acid in patients with primary biliary cirrhosis and inadequate response to ursodeoxycholic acid. Gastroenterology 2015;148:751-761.e8.

47 Anwer MS, Stieger B: Sodium-dependent bile salt transporters of the SLC10A transporter family: more than solute transporters. Pflugers Arch 2014;466:77-89.

-48 Zhang Y, Csanaky IL, Cheng X, et al: Organic anion transporting polypeptide 1a1 null mice are sensitive to cholestatic liver injury. Toxicol Sci 2012;127:451-462.

49 Kuiper EM, van Erpecum KJ, Beuers U, et al: The potent bile acid sequestrant colesevelam is not effective in cholestatic pruritus: results of a double-blind, randomized, placebo-controlled trial. Hepatology 2010;52:1334-1340.

50 Graffner H, Gillberg PG, Rikner L, et al: The ileal bile acid transporter inhibitor A4250 decreases serum bile acids by interrupting the enterohepatic circulation. Aliment Pharmacol Ther 2016;43:303-310.

51 Baghdasaryan A, Fuchs CD, Österreicher CH, et al: Inhibition of intestinal bile acid absorption improves cholestatic liver and bile duct injury in a mouse model of sclerosing cholangitis. J Hepatol 2016;64:674-681.

52 Miethke AG, Zhang W, Simmons J, et al: Pharmacological inhibition of apical sodiumdependent bile acid transporter changes bile composition and blocks progression of sclerosing cholangitis in multidrug resistance 2 knockout mice. Hepatology 2016;63:512523.

53 Inagaki T, Choi M, Moschetta A, et al: Fibroblast growth factor 15 functions as an enterohepatic signal to regulate bile acid homeostasis. Cell Metab 2005;2:217-225.
Bile Acid Uptake Transporters as Targets for Therapy
Dig Dis 2017;35:251-258 
54 Modica S, Petruzzelli M, Bellafante E, et al: Selective activation of nuclear bile acid receptor FXR in the intestine protects mice against cholestasis. Gastroenterology 2012;142:355365.e1-e4.

55 Luo J, Ko B, Elliott M, et al: A nontumorigenic variant of FGF19 treats cholestatic liver diseases. Sci Transl Med 2014;6:247ra100.

56 Zhou M, Learned RM, Rossi SJ, et al: Engineered fibroblast growth factor 19 reduces liver injury and resolves sclerosing cholangitis in Mdr2-deficient mice. Hepatology 2016;63: 914-929.

57 Mayo MJ, Wigg AJ, Roberts SK, Arnold H, et al: NGM282, a novel variant of FGF-19, demonstrates biologic activity in primary biliary cirrhosis patients with an incomplete response to ursodeoxycholic acid:results of a phase 2 multicenter, randomized, double blinded, placebo controlled trial. Hepatology 2015;62:263A-264A.

-58 Schaap FG, Trauner M, Jansen PL: Bile acid receptors as targets for drug development. Nat Rev Gastroenterol Hepatol 2014;11:5567.

59 de Aguiar Vallim TQ, Tarling EJ, Edwards PA. Pleiotropic roles of bile acids in metabolism. Cell Metab 2013;17:657-669.

60 Rudling M, Camilleri M, Graffner H, et al: Specific inhibition of bile acid transport alters plasma lipids and GLP-1. BMC Cardiovasc Disord 2015;15:75.

61 Lundåsen T, Andersson EM, Snaith M, et al: Inhibition of intestinal bile acid transporter Slc10a2 improves triglyceride metabolism and normalizes elevated plasma glucose levels in mice. PLoS One 2012;7:e37787.

62 Rao A, Kosters A, Mells J, et al: Administration of an ileal apical sodium-dependent bile acid transporter inhibitor protects against non-alcoholic fatty liver disease in high fat diet-fed mice. Hepatology 2015;62:217A.
63 Harach T, Pols TW, Nomura M, Maida A, et al: TGR5 potentiates GLP-1 secretion in response to anionic exchange resins. Sci Rep 2012;2:430.

64 Potthoff MJ, Potts A, He T, et al: Colesevelam suppresses hepatic glycogenolysis by TGR5mediated induction of GLP-1 action in DIO mice. Am J Physiol Gastrointest Liver Physiol 2013;304:G371-G380.

65 Nwose OM, Jones MR: Atypical mechanism of glucose modulation by colesevelam in patients with type 2 diabetes. Clin Med Insights Endocrinol Diabetes 2013;6:75-79.

66 Ullmer C, Alvarez Sanchez R, Sprecher U, et al: Systemic bile acid sensing by $G$ proteincoupled bile acid receptor 1 (GPBAR1) promotes PYY and GLP-1 release. Br J Pharmacol 2013;169:671-684.

67 Brighton CA, Rievaj J, Kuhre RE, et al: Bile acids trigger GLP-1 release predominantly by accessing basolaterally located G proteincoupled bile acid receptors. Endocrinology 2015;156:3961-3970.

68 Thomas C, Gioiello A, Noriega L, et al: TGR5mediated bile acid sensing controls glucose homeostasis. Cell Metab 2009;10:167-177.

69 Watanabe M, Houten SM, Mataki C, et al: Bile acids induce energy expenditure by promoting intracellular thyroid hormone activation. Nature 2006;439:484-489.

70 Pournaras DJ, Glicksman C, Vincent RP, et al: The role of bile after Roux-en-Y gastric bypass in promoting weight loss and improving glycaemic control. Endocrinology 2012;153: 3613-3619.

71 Cummings BP, Bettaieb A, Graham JL, et al: Vertical sleeve gastrectomy improves glucose and lipid metabolism and delays diabetes onset in UCD-T2DM rats. Endocrinology 2012; 153:3620-3632.

72 Kohli R, Kirby M, Setchell KD, et al: Intestinal adaptation after ileal interposition surgery increases bile acid recycling and protects against obesity-related comorbidities. Am J Physiol Gastrointest Liver Physiol 2010;299:G652G660.
Ryan KK, Tremaroli V, Clemmensen C, et al: FXR is a molecular target for the effects of vertical sleeve gastrectomy. Nature 2014;509: 183-188.

74 Ding L, Sousa KM, Jin L, Dong B, et al: Vertical sleeve gastrectomy activates GPBAR-1/ TGR5 to sustain weight loss, improve fatty liver, and remit insulin resistance in mice. Hepatology 2016;64:760-773.

75 Myronovych A, Kirby M, Ryan KK, et al: Vertical sleeve gastrectomy reduces hepatic steatosis while increasing serum bile acids in a weight-loss-independent manner. Obesity (Silver Spring) 2014;22:390-400.

76 Keitel V, Reinehr R, Gatsios P, et al: The Gprotein coupled bile salt receptor TGR5 is expressed in liver sinusoidal endothelial cells. Hepatology 2007;45:695-704.

77 Kawamata Y, Fujii R, Hosoya M, et al: A G protein-coupled receptor responsive to bile acids. J Biol Chem 2003;278:9435-9440.

78 Calmus Y, Guechot J, Podevin P, et al: Differential effects of chenodeoxycholic and ursodeoxycholic acids on interleukin 1, interleukin 6 and tumor necrosis factor-alpha production by monocytes. Hepatology 1992;16: 719-723.

79 Pols TW, Nomura M, Harach T, et al: TGR5 activation inhibits atherosclerosis by reducing macrophage inflammation and lipid loading. Cell Metab 2011;14:747-757.

80 Vassileva G, Hu W, Hoos L, Tetzloff G, et al: Gender-dependent effect of Gpbar1 genetic deletion on the metabolic profiles of diet-induced obese mice. J Endocrinol 2010;205: 225-232.

-81 Rose AJ, Berriel Díaz MB, Reimann A, et al: Molecular control of systemic bile acid homeostasis by the liver glucocorticoid receptor. Cell Metab 2011;14:123-130.

82 Oehler N, Volz T, Bhadra OD, et al: Binding of hepatitis $\mathrm{B}$ virus to its cellular receptor alters the expression profile of genes of bile acid metabolism. Hepatology 2014;60:1483-1493. 